\title{
AJK2015-XXXXX
}

\section{A DYNAMICALLY ADAPTIVE LATTICE BOLTZMANN METHOD FOR FLAPPING WING AERODYNAMICS}

\author{
Stephen L. Wood \\ University of Tennessee - Knoxville \\ The Bredesen Center \\ 821 Volunteer Blvd., Knoxville, TN 37996, USA
}

\author{
Ralf Deiterding \\ German Aerospace Center (DLR) \\ Institute of Aerodynamics and Flow Technology \\ Bunsenstr. 10, 37073 Göttingen, Germany
}

\begin{abstract}
The essential components of a parallel dynamically adaptive lattice Boltzmann method coupled to a 6-degree-offreedom rigid body motion solver are presented. This Cartesian approach with automatic fluid meshing is particularly well suited for simulating the interaction of low Reynolds number flows and moving structures with good accuracy and high computational performance. The fully coupled fluid-structure simulation method is demonstrated for the experiment of a twosegment hinged wing with torsion damper by Toomey \& Eldredge, a simplistic model of a flapping wing in air. A grid convergence study assesses the prediction accuracy of the overall method and required CPU times. Our computations show very good agreement with measurements of the evolving hinge angle by Toomey \& Eldredge; forces and moments are predicted with an error margin of generally $<5 \%$ with respect to their computational results.
\end{abstract}

\section{NOMENCLATURE}

$C, T$ Collision and transport operator of Lattice Boltzmann method

$c_{s}, a \quad$ Physical speed of sound, lattice speed

$\mathbf{e}_{\alpha} \quad$ Set of lattice velocity vectors

$f, f^{e q}$ Distribution and equilibrium distribution function

$f_{\alpha}, f_{\alpha}^{e q}$ Discrete distribution functions

$f_{\alpha}^{f}, f_{\alpha}^{C}$ Discrete distributions on next finer and coarser level

$p \quad$ Dynamic fluid pressure

$\mathbf{u}, u_{i} \quad$ Fluid velocity vector and ith velocity component

w Wall velocity

$\mathbf{x}, \Delta x \quad$ Point in space, mesh spacing

$t, \Delta t \quad$ Time, discrete time step

$\rho \quad$ Fluid density

$v \quad$ Viscosity

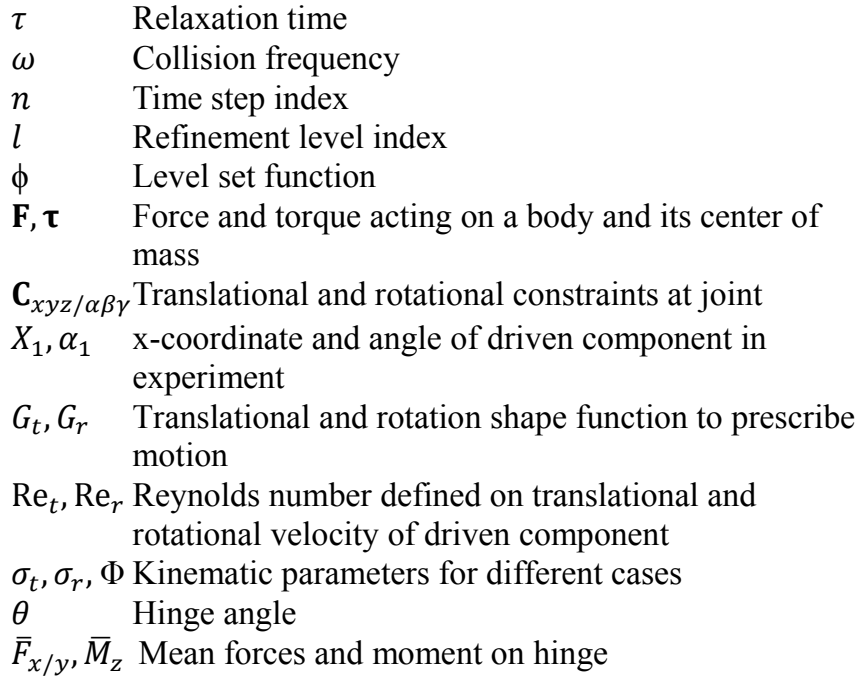

\section{INTRODUCTION}

The aerodynamics of flapping wings are characterized by a complex interaction between moving structures and fluid flow. Unstructured finite volume methods that use grids following the motion have to deal with difficult mesh morphing, untangling and possibly grid regeneration and data remapping problems. As an alternative to the usually employed implicit, typically pressure-correction based CFD solution algorithms, we adopt in here the lattice Boltzmann method (LBM), cf. Aono et al. [1]. The LBM is based on solving the Boltzmann equation in a specially chosen, discrete phase space and fully explicit in time [2]. The LBM is constructed on uniform Cartesian grids and geometrically complex boundaries are considered with an immersed boundary approach, making the method well suited for considering moving structures. Here, we 
utilize a level set distance function to represent embedded objects. Dynamic mesh adaptation is applied in addition to increase the local resolution based on the level set function and features detected in the flow field [3]. Distributed memory parallelization is adopted to allow for large-scale simulations.

The paper is organized as follows: We first recall the construction principles of the LBM. In the next section, the block-based mesh adaptation procedure and in particular the incorporation of the LBM are presented. The third section explains our approach in dealing with embedded geometries in the LBM and how we compute rigid body dynamics. Finally, validation results of a two-segment hinged wing with torsion damper modeling a simplistic flapping wing as proposed by Toomey \& Eldredge [4] are presented and discussed. The computations confirm the benefit of the proposed overall approach for flapping wing dynamics and biologically inspired fluid-structure interaction problems.

\section{LATTICE BOLTZMANN METHOD}

The lattice Boltzmann method is based on computing approximations of the Boltzmann equation with a simplified collision operator

$$
\partial_{t} f+\mathbf{u} \cdot \nabla f=\omega\left(f^{e q}-f\right)
$$

on a rectangular grid of characteristic domain length $L$ with isotropic mesh spacing $\Delta x$ under the assumption of a small Knudsen number $K n=l_{f} / L \ll 1$, where the mean free path length $l_{f}$ is replaced with $\Delta x$. A crucial idea of the LBM is to approximate Eq. (1) in a specially chosen discrete phase space, in which a particle distribution function $f_{\alpha}(\mathbf{x}, t)$ is associated to every discrete lattice velocity $\mathbf{e}_{\alpha}$. The total density distribution is given as $\rho(\mathbf{x}, t)=\sum_{\alpha} f_{\alpha}(\mathbf{x}, t)$ and the macroscopic moments as $\rho(\mathbf{x}, t) u_{i}(\mathbf{x}, t)=\sum_{\alpha} \mathbf{e}_{\alpha i} f_{\alpha}(\mathbf{x}, t)$. A splitting approach is then adopted that first solves the homogeneous transport equation with the time-explicit update step

$$
T: \quad \tilde{f}_{\alpha}\left(\mathbf{x}+\mathbf{e}_{\alpha} \Delta t, t+\Delta t\right)=f_{\alpha}(\mathbf{x}, t) .
$$

Here, we apply the D3Q19 model for which the lattice velocities are defined as

$$
\mathbf{e}_{\alpha}=\left\{\begin{array}{cc}
0, & \alpha=0, \\
( \pm 1,0,0) a,(0, \pm 1,0) a,(0,0, \pm 1) a, & \alpha=1, \ldots, 6, \\
( \pm 1, \pm 1,0) a,( \pm 1,0, \pm 1) a,(0, \pm 1, \pm 1) a, & \alpha=7, \ldots, 18,
\end{array}\right.
$$

with $a=\Delta x / \Delta t$. The physical speed of sound $c_{s}$ is related to $a$ by $c_{s}=a / \sqrt{3}$. The right-hand of Eq. (1) is integrated subsequently by the collision operator

$$
\begin{aligned}
C: \quad f_{\alpha}(\mathbf{x}, t+\Delta t)=\tilde{f}_{\alpha}(\mathbf{x}, t+\Delta t)+ \\
\omega \Delta t\left(\tilde{f}_{\alpha}^{e q}(\mathbf{x}, t+\Delta t)-\tilde{f}_{\alpha}(\mathbf{x}, t+\Delta t)\right),
\end{aligned}
$$

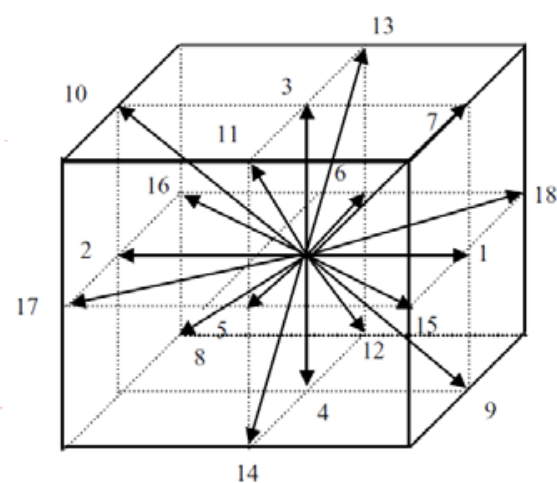

FIGURE 1. THE VELOCITIES $\mathbf{e}_{\alpha}$ OF THE D3Q19 LATTICE.

with equilibrium function

$$
f_{\alpha}^{e q}(\rho, \mathbf{u})=\rho t_{\alpha}\left[1+\frac{3 e_{\alpha} \mathbf{u}}{a^{2}}+\frac{9\left(e_{\alpha} \mathbf{u}\right)^{2}}{2 a^{4}}-\frac{3 \mathbf{u}^{2}}{2 a^{2}}\right],
$$

with $t_{0}=1 / 3, t_{\alpha}=1 / 18$ for $\alpha=1, \ldots, 6$ and $t_{\alpha}=1 / 36$ for $\alpha=7, \ldots, 18$. The variation in hydrodynamic pressure for the equilibrium function (5) reads $p=\sum_{\alpha} f_{\alpha}^{e q} c_{s}^{2}=\left(\rho-\rho_{0}\right) c_{s}^{2}$.

Applying a Chapman-Enskog expansion procedure, it can be shown [5] that the sketched LBM converges to a solution of the weakly compressible Navier-Stokes equations

$$
\begin{gathered}
\partial_{t} \rho+\nabla \cdot(\rho \mathbf{u})=0, \\
\partial_{t} \mathbf{u}+\mathbf{u} \cdot \nabla \mathbf{u}=-\nabla p+v \nabla^{2} \mathbf{u} .
\end{gathered}
$$

It can be shown further, cf. [2], that the kinematic viscosity $v$ and collision frequency $\omega$ are connected by the relation

$$
\omega=\tau^{-1}=\frac{c_{s}^{2} \Delta t}{v+\Delta t c_{s}^{2} / 2} .
$$

While the sketched model can be used directly to simulate laminar flows, it is mandatory to apply a turbulence model in addition in high Reynolds number situations. In the context of LBM, it is most common to adopt a large eddy simulation approach, cf. [5]

\section{DYNAMIC MESH ADAPTATION}

For local dynamic mesh adaptation we have adopted the block-structured adaptive mesh refinement (SAMR) method after Berger \& Collela [6]. In order to fit smoothly into our existing, fully parallelized finite volume SAMR software system AMROC [3], we have implemented the LBM cellbased, which makes the scheme also conservative in $\rho$ and $\rho u_{i}$. In the SAMR approach, finite volume cells are clustered with a special algorithm into non-overlapping rectangular grids. The grids have a suitable layer of halo cells for synchronization 
and applying inter-level and physical boundary conditions. Refinement levels are integrated recursively. The spatial mesh width $\Delta x_{l}$ and the time step $\Delta t_{l}$ are refined by the same factor $r_{l}$,where we assume $r_{l} \geq 2$ for $l>0$ and $r_{0}=1$.

Note that in an adaptive LBM the collision frequency $\omega_{l}$ is not a constant but needs to be adjusted according to Eq. (8) for the update on each level. In addition to this, the interface region requires a specialized treatment. Distinguishing between the transport and collision operators, $T$ and $C$, cf. Eqs. (2) and (4), the steps of our method for a refinement factor of 2 are:

1. Complete update on coarse grid: $f_{\alpha}^{C, n+1}:=C T\left(f_{\alpha}^{C, n}\right)$

2. Use coarse grid distributions $f_{\alpha, i n}^{C, n}$ that propagate into the fine grid, cf. Fig. 2(a), to construct initial fine grid halo values $f_{\alpha, \text { in }}^{f, n}$, cf. Fig. 2(b).

3. Complete transport $\tilde{f}_{\alpha}^{f, n}:=T\left(f_{\alpha}^{f, n}\right)$ on whole fine mesh. Collision $f_{\alpha}^{f, n+1 / 2}:=C\left(\tilde{f}_{\alpha}^{f, n}\right)$ is applied only in the interior cells (yellow in Fig. 2(b)).

4. Repeat 3. to obtain $\tilde{f}_{\alpha}^{f, n+1 / 2}:=T\left(f_{\alpha}^{f, n+1 / 2}\right)$ and $f_{\alpha}^{f, n+1}:=C\left(f_{\alpha}^{f, n+1 / 2}\right)$

5. Average outgoing distributions from fine grid halos (Fig. 2(c)), that is $f_{\alpha, \text { out }}^{f, n+1 / 2}$ in the inner halo layer and $\tilde{f}_{\alpha, \text { out }}^{f, n}$ (outer halo layer) to obtain $\tilde{f}_{\alpha, \text { out }}^{C, n}$.

6. Revert transport for averaged outgoing distributions, $\tilde{f}_{\alpha, \text { out }}^{C, n}:=T^{-1}\left(\tilde{f}_{\alpha, \text { out }}^{C, n}\right)$, and overwrite those in the previous coarse grid time step, cf. Fig. 2(d).
7. Parallel synchronization of $\tilde{f}_{\alpha}^{C, n}, \tilde{f}_{\alpha, \text { out }}^{C, n}$ on entire level.

8. Repeat complete update on coarse grid cells next to coarsefine boundary only: $\tilde{f}_{\alpha, \text { out }}^{C, n}:=T^{-1}\left(\tilde{f}_{\alpha, \text { out }}^{C, n}\right)$

This algorithm is computationally equivalent to the method by Chen et al. [7] but tailored to the SAMR recursion that updates coarse grids in their entirety before fine grids are computed. Because of the nonlinearity of the collision operator $C$ it becomes necessary under this paradigm to repeat the LBM update for those coarse grid cells that share a face or corner with a fine grid.

\section{EMBEDDED STRUCTURE HANDLING}

We represent non-Cartesian boundaries implicitly on the adaptive Cartesian grid by utilizing a scalar level set function $\phi$ that stores the distance to the boundary surface. The boundary surface it located exactly at $\phi=0$ and the boundary outer normal in every mesh point can be evaluated as $\mathbf{n}=-\nabla \phi /|\nabla \phi|[8]$. We treat a fluid cell as an embedded ghost cell if its midpoint satisfies $\phi<0$.

In order to implement non-Cartesian boundary conditions with the LBM, we have chosen to pursue a 1st order accurate ghost fluid approach that was already available in AMROC [4]. In our technique, the density distributions in embedded ghost cells are adjusted to model the boundary conditions of a nonCartesian reflective wall moving with velocity $\mathbf{w}$ before applying the unaltered LBM. The last step involves interpolation and mirroring of $\rho, \mathbf{u}$ across the boundary to $\rho^{\prime}$ and $\overline{\mathbf{u}}$ and modification of the macro velocity in the immersed boundary cells to $\mathbf{u}^{\prime}=2 \mathbf{w}-\overline{\mathbf{u}}$, cf. [3]. From the newly constructed macroscopic values the density distributions in the embedded ghost cells are simply set to $f_{\alpha}^{e q}\left(\rho^{\prime}, \mathbf{u}^{\prime}\right)$.

Real-world geometries are considered in AMROC as triangular surface meshes. The computation of the level set distance information in every Cartesian mesh point could (a)

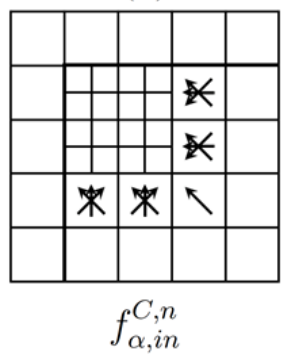

(b)

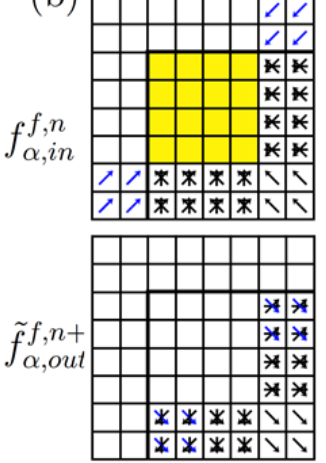

(c)

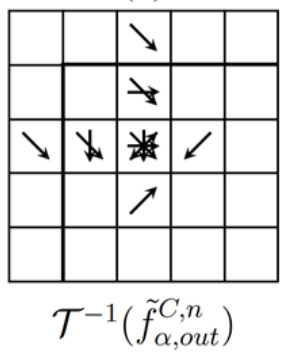

FIGURE 2. DISTRIBUTIONS INVOLVED IN NECESSARY DATA EXCHANGE AT A COARSE-FINE BOUNDARY. (A) COARSE DISTRIBUTIONS GOING INTO FINE GRID; (B) INGOING INTERPOLATED FINE DISTRIBUTIONS IN HALOS (TOP), OUTGOING DISTRIBUTIONS IN HALOS AFTER TWO FINE-LEVEL TRANSPORT STEPS (BOTTOM); (C) AVERAGED DISTRIBUTIONS REPLACING COARSE VALUES BEFORE UPDATE IS REPEATED IN CELLS NEXT TO BOUNDARY. 
principally be accomplished by simply iterating over the entire surface mesh; yet, this would lead to detrimental performance for increasing mesh size. The problem is equivalent to determining for every Cartesian cell the closest facet on the surface mesh. For this purpose, we employ a specially developed algorithm based on characteristic reconstruction and scan conversion developed by Mauch [9] that is used to compute the distance exactly only in a small band around the embedded structure.

The dynamics of multi-body systems undergoing interaction with the fluid are modeled as sets of triangulated surface meshes configured in kinetic chains. The dynamics of these mechanisms are solved by a recursive Newton-Euler method at each time step [10]. Considering an arbitrary link with a coordinate frame located at point $\mathbf{P}$ that is not coincident with its associated body's center of mass, the force and torque applied by the preceding link are

$$
\begin{aligned}
&\left(\begin{array}{c}
\mathbf{F} \\
\boldsymbol{\tau}_{P}
\end{array}\right)=\left(\begin{array}{cc}
m \mathbf{1} & -m[\mathbf{c}]^{\times} \\
m[\mathbf{c}]^{\times} \mathbf{I}_{c m} & -m[\mathbf{c}]^{\times}[\mathbf{c}]^{\times}
\end{array}\right)\left(\begin{array}{c}
\mathbf{a}_{p} \\
\boldsymbol{\alpha}
\end{array}\right)+ \\
&\left(\begin{array}{c}
m[\boldsymbol{\omega}]^{\times}[\boldsymbol{\omega}]^{\times} \mathbf{c} \\
{[\boldsymbol{\omega}]^{\times}\left(\mathbf{I}_{c m}-m[\mathbf{c}]^{\times}[\mathbf{c}]^{\times}\right) \boldsymbol{\omega}}
\end{array}\right),
\end{aligned}
$$

where $m$ is the mass of the body, $\mathbf{1}$ denotes the $4 \times 4$ identity matrix, $\mathbf{a}_{P}$ is the acceleration of link frame with origin at $\mathbf{P}$ in the preceding link's frame. $\mathbf{I}_{c m}$ is the moment of inertia about the center of mass, $\boldsymbol{\omega}$ and $\boldsymbol{\alpha}$ are angular velocity and acceleration, respectively, $\mathbf{c}$ is the location of the body's center of mass expressed in the associated link's frame, and $[\mathbf{c}]^{\times},[\boldsymbol{\omega}]^{\times}$ denote skew-symmetric cross product matrices.

Here, we additionally define the total force and torque acting on a body, $\mathbf{F}=\left(\mathbf{F}_{F S I}+\mathbf{F}_{\text {prescribed }}\right) \cdot \mathbf{C}_{x y z}$ and $\boldsymbol{\tau}=\left(\boldsymbol{\tau}_{F S I}++\boldsymbol{\tau}_{\text {Prescribed }}\right) \cdot \mathbf{C}_{\alpha \beta \gamma}$ respectively. Where $\mathbf{C}_{x y z}$ and $\mathbf{C}_{\alpha \beta \gamma}$ are the translational and rotational constraints, respectively. $\mathbf{F}_{F S I}$ and $\boldsymbol{\tau}_{F S I}$ are determined for each body by integrating the fluid hydrodynamic pressure and viscous forces on the triangular facets of the respective body's surface mesh. Each surface mesh (lofted from .xyz curves or loaded from .obj, .ply, .stl formats) is associated with a kinetic link in a chain that begins with a base link in the global coordinate frame. Links are connected by joints that may be independently constrained in six degrees of freedom relative to the preceding link. The evolution of the triangular surface mesh as well as the velocity $\mathbf{w}$ in each node are communicated to the LBM fluid solver in dedicated coupling time steps. The data exchange corresponds to the time step of an SAMR level but this does not have to be the finest refinement level available, cf. [11]. This formulation readily facilitates the kinetic analysis of each link and triangulated surface in the global coordinate frame or in any of the link coordinate frames.

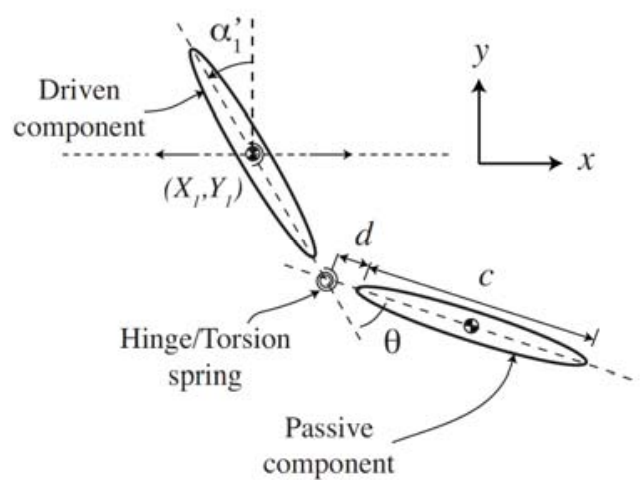

FIGURE 3. MODEL SYSTEM CONSISTING OF TWO RIGID ELLIPTICAL SECTIONS CONNECTED BY A HINGE WITH TORSION SPRING AND DAMPER.

\section{RESULTS}

A canonical problem of fluid-structure interaction and wake prediction proposed by Toomey \& Eldredge [4] is selected as a validation test case for coupled flapping wing aerodynamics. This model, depicted in Fig. 3, utilizes a system of two articulated rigid bodies connected by a torsion spring and damper. The kinematics of the centroid of the driven wing are prescribed, while the trailing body responds passively to the aerodynamic and inertial/elastic forces. The principle unknown in this rigid body dynamics problem is the hinge angle, $\theta$. The parametric kinematic equations

$$
\begin{gathered}
X_{1}(t)=\frac{A_{0}}{2} \frac{G_{t}(f t)}{\max G_{t}}, \quad \alpha_{1}(t)=-\beta \frac{G_{r}(f t)}{\max G_{r}}, \\
C(t)=\frac{\tanh (8 t-2)+\tanh (2)}{t+\tanh (2)}, \\
G_{t}(t)=\int_{t} \tanh \left(\sigma_{t} \cos \left(2 \pi t^{\prime}\right)\right) d t^{\prime}, \\
G_{r}(t)=\tanh \left(\sigma_{r} \cos (2 \pi t+\Phi)\right)
\end{gathered}
$$

describe the motion of the driven body. The parameters utilized in this work and in [1] to specify the kinematics through the translational, $G_{t}(t)$, and rotational, $G_{r}(t)$, shape functions are given in Table 1. The start-up conditioner, $C_{t}(t)$, is applied to the translational kinematics to avoid an impulsive start. The translational and rotational Reynolds numbers are based on the peak translational, $V$, and rotational, $2 \pi \beta \sigma_{r} f c \tanh \left(\sigma_{r}\right)$, velocities as shown in

$$
\operatorname{Re}_{t}=V c / v, \quad \operatorname{Re}_{r}=2 \pi \beta \sigma_{r} f c^{2} /\left(\tanh \left(\sigma_{r}\right) v\right) .
$$

The used torsion spring and damper coefficients are $K^{*}=6.9 \cdot 10^{-3} \mathrm{~kg} \mathrm{~m}^{2} / \mathrm{s}^{2}$ and $R^{*}=3.8 \cdot 10^{-4} \mathrm{~kg} \mathrm{~m}^{2} / \mathrm{s}^{2}$ respectively. A no-slip boundary condition is applied at the wing surface and at the top and bottom (y direction) boundaries. The simulation domain is a box of extents 
TABLE 1. KINEMATIC PARAMETERS

\begin{tabular}{llcl}
$A_{0}(\mathrm{~cm})$ & 7.1 & $\Phi$ & 0,45 \\
$c(\mathrm{~cm})$ & 5.1 & $R e_{t}$ & 73,370 \\
$d(\mathrm{~cm})$ & 0.25 & $R e_{r}$ & 100,500 \\
$\beta$ & $\pi / 4$ & $\rho_{b}\left(\mathrm{~kg} / \mathrm{m}^{3}\right)$ & 5080 \\
$\sigma_{t}$ & $0.628,1.885,3.770$ & $f(\mathrm{~Hz})$ & 0.15 \\
$\sigma_{r}$ & $0.628,1.885,3.770$ & & \\
\hline
\end{tabular}

TABLE 2. CONVERGENCE STUDY PARAMETERS

\begin{tabular}{ccccc}
\hline Case & 2.1 & 2.2 & 2.3 & 2.4 \\
\hline \multicolumn{1}{c}{$|\nabla \vec{v}|$} & 0.5 & 0.5 & 0.5 & 0.5 \\
net refine & 0.125 & 0.0625 & 0.0625 & 0.0625 \\
finest $\Delta x / c$ & $2.45 \mathrm{E}-02$ & $1.22 \mathrm{E}-02$ & $6.12 \mathrm{E}-03$ & $3.06 \mathrm{E}-03$ \\
finest $\Delta t / \tau_{r}$ & $3.53 \mathrm{E}-04$ & $8.83 \mathrm{E}-05$ & $4.42 \mathrm{E}-05$ & $2.21 \mathrm{E}-05$ \\
\hline
\end{tabular}

$x:[-0.5,0.5], y:[-0.5,0.5], z:[-0.31,0.31]$. It is periodic in the $x$-direction and has slip walls at the $z$-boundaries to minimize corner effects similar to the confguration used by Aono et al. [1].

A convergence study was conducted for the moderate kinematic parameters $\left(\sigma_{t}=1.85, \sigma_{r}=1.885, \Phi=0\right)$ assigned to Case 2 for the simulation parameters given in Table 2 . The error in predicted hinge deflection relative to the experiments conducted by Toomey \& Eldredge for each of the convergence study cases is presented in Table 3 along with the wall time of each simulation on 24 Intel-Ivybridge CPUs. The errors in mean and peak forces and moments relative to the values predicted by VVPM [4] are shown in Tables 4 and 5 respectively. The spatial and temporal resolution at the wing surface in Case 2.2 predicts peak hinge deflection, forces and moment accurately at moderate computational cost. These parameters where selected to simulate the seven kinematic cases investigated by Toomey \& Eldredge [4]. Eddies shed by the moving wing in Case 2 are clearly depicted in the vorticity field at two times in Fig. 4. Regions of mesh refinement are shown in Fig. 5 and the domain decomposition is displayed in Fig. 6 plainly presenting the adaptive refinement and load balancing during runtime within AMROC.

Figures 7-9 display the hinge deflection angle for experiments and our simulations through three periods of motion for Cases 1, 2 and 4. The mean and peak fluid loads are simulated in this work are within $5 \%$ of those predicted by the VVPM [4] as shown in Tabs. 6 and 7. Hinge deflections presented in Figs. 7-9 are in good agreement with the experimental results [4]. Comparing Cases 1, 2, and 4, where the translational and rotational shape parameters are increased simultaneously the expected increases in deflection angle, mean and peak forces and moment are observed. Figure 9 clearly depicts the expected large deflection opposite the initial rotation followed by a recoil. In contrast, steady translation causes a small aft rotation. Comparing Cases 4 and 6 the expected decrease in hinge deflection corresponds to the reduced rotation rate caused by the rotational shape parameter, $\sigma_{r}$. The insensitivity of hinge deflection to translation rate controlled by $\sigma_{t}$ is shown in the comparison of Cases 4 and 7. The deviation at small hinge angles observed in Case 7 (Fig. 9) corresponds to periods of the flaping cycle when the driven lead wing component is translating and rotating very slowly and the trailing wing component is recoiling. In the experiments the hinge was observed to behave nonlinearly at small angles and under small loads. This nonlinearity was not accounted for in this work or in the simulations by Aono et al. [1] or those by Toomey \& Eldredge $[4,12]$ to similar effect.

TABLE 3. CONVERGENCE STUDY RELATIVE ERROR OF MEAN AND PEAK HINGE DEFLECTION VS WALL TIME

\begin{tabular}{ccccccc}
\hline & \multicolumn{3}{c}{$\operatorname{Re}_{r}=100$} & \multicolumn{3}{c}{$\operatorname{Re}_{r}=500$} \\
Case & Mean & Peak & Wall [s] & Mean & Peak & Wall [s] \\
\hline Ref. & $-0.124^{\circ}$ & $17.167^{\circ}$ & - & $-0.116^{\circ}$ & $17.980^{\circ}$ & - \\
2.1 & $-8.31 \%$ & $-0.95 \%$ & 625 & $-8.08 \%$ & $-0.93 \%$ & 656 \\
2.2 & $-8.31 \%$ & $-0.81 \%$ & 2047 & $-8.01 \%$ & $-0.79 \%$ & 2118 \\
2.3 & $-2.11 \%$ & $-0.03 \%$ & 7630 & $-2.08 \%$ & $-0.03 \%$ & 7974 \\
2.4 & $-2.01 \%$ & $-0.01 \%$ & 43318 & $-2.00 \%$ & $-0.01 \%$ & 44834 \\
\hline
\end{tabular}

TABLE 4. CONVERGENCE STUDY RELATIVE ERROR OF NONDIMENSIONAL MEAN FORCE AND MOMENTS

\begin{tabular}{ccccccc}
\hline & \multicolumn{3}{c}{$R e_{r}=100$} & \multicolumn{3}{c}{$\operatorname{Re}_{r}=500$} \\
Case & $\bar{F}_{x}$ & $\bar{F}_{y}$ & $\bar{M}_{z}$ & $\bar{F}_{x}$ & $\bar{F}_{y}$ & $\bar{M}_{z}$ \\
\hline Ref. & -7 & 58.8 & -4 & -4.9 & 81.6 & -2 \\
2.1 & $5.47 \%$ & $3.74 \%$ & $5.55 \%$ & $5.35 \%$ & $6.83 \%$ & $-7.29 \%$ \\
2.2 & $2.47 \%$ & $0.74 \%$ & $2.55 \%$ & $2.35 \%$ & $3.83 \%$ & $-4.29 \%$ \\
2.3 & $2.22 \%$ & $0.49 \%$ & $2.30 \%$ & $2.10 \%$ & $3.58 \%$ & $-4.04 \%$ \\
2.4 & $2.17 \%$ & $0.44 \%$ & $2.25 \%$ & $2.05 \%$ & $3.53 \%$ & $-3.99 \%$ \\
\hline
\end{tabular}

TABLE 5. CONVERGENCE STUDY RELATIVE ERROR OF NONDIMENSIONAL PEAK FORCE AND MOMENTS

\begin{tabular}{ccccccc}
\hline & \multicolumn{3}{c}{$\operatorname{Re}_{r}=100$} & \multicolumn{3}{c}{$\operatorname{Re}_{r}=500$} \\
Case & $\bar{F}_{x}$ & $\bar{F}_{y}$ & $\bar{M}_{z}$ & $\bar{F}_{x}$ & $\bar{F}_{y}$ & $\bar{M}_{z}$ \\
\hline Ref. & 876 & 359 & 397 & 863 & 373 & 382 \\
2.1 & $7.81 \%$ & $5.77 \%$ & $5.97 \%$ & $6.07 \%$ & $7.68 \%$ & $-5.69 \%$ \\
2.2 & $4.46 \%$ & $2.42 \%$ & $2.62 \%$ & $2.72 \%$ & $4.33 \%$ & $-2.34 \%$ \\
2.3 & $4.12 \%$ & $2.08 \%$ & $2.28 \%$ & $2.38 \%$ & $3.99 \%$ & $-2.00 \%$ \\
2.4 & $4.08 \%$ & $2.04 \%$ & $2.24 \%$ & $2.34 \%$ & $3.95 \%$ & $-1.96 \%$ \\
\hline
\end{tabular}


The influence of rotational phase is observed by comparing Cases 2 and 3, as well as, 4 and 5. In both comparisons the mean $y$-force is slightly increased and hinge deflection is only changed by a phase shift. These simulations show that the rate of rotation of the driven body is the major cause of hinge deflection as was found in the experiments conducted by Toomey \& Eldredge $[4,12]$.

\section{CONCLUSIONS}

The first prototype of a dynamically adaptive, threedimensional lattice-Boltzmann method for simulation of flapping wing dynamics has been developed. First validation has been achieved for a canonical FSI problem from [4]. We have confirmed that our approach is able to simulate the propagation of wake fields created by the translation and rotation of simplified hinged wing geometry, including the interaction with previously shed vortices, with apparent good quality and comparably moderate computational costs. Immediate future work will concentrate on incorporating the dynamic elastic response of the components into simplified biologically relevant models and validating the approach for available benchmarks.

\section{ACKNOWLEDGMENTS}

Stephen L. Wood was supported by the TN-SCORE Energy Scholar program funded by NSF EPS-1004083 during this work.

\section{REFERENCES}

[1] H. Aono, A. Gupta, D. Qi, Wei Shyy. The Lattice Boltzmann Method for Flapping Wing Aerodynamics. AIAA-2010-4867, 40th Fluid Dynamics Conference and Exhibit, Chicago, Illinois, June 28-1, 2010.

[2] D. Hähnel. Molekulare Gasdynamik. Springer, 2004.

[3] R. Deiterding. Block-structured adaptive mesh refinement - theory, implementation and application. European Series in Applied and Industrial Mathematics: Proceedings, 34:97-150, 2011.

[4] J. Toomey and J. D. Eldredge. Numerical and experimental study of the fluid dynamics of a flapping wing with low order flexibility. Physics of Fluids (1994present), 20(7):-, 2008.

[5] S. Hou, J. Sterling, S. Chen, and G. D. Doolen. A lattice Boltzmann subgrid model for high Reynolds number flows. In A. T. Lawniczak and R. Kapral, editors, Pattern formation and lattice gas automata, volume 6, pages 151166. Fields Inst Comm, 1996.

[6] M. Berger and P. Colella. Local adaptive mesh refinement for shock hydrodynamics. J. Comput. Phys., 82:64-84, 1988.

[7] H. Chen, O. Filippova, J. Hoch, K. Molvig, R. Shock, C. Teixeira, and R. Zhang. Grid refinement in lattice Boltzmann methods based on volumetric formulation. Physica A, 362:158-167, 2006.
[8] R. Deiterding. A parallel adaptive method for simulating shock-induced combustion with detailed chemical kinetics in complex domains. Computers \& Structures, 87:769783, 2009.

[9] S. P. Mauch. Efficient Algorithms for Solving Static Hamilton-Jacobi Equations. $\mathrm{PhD}$ thesis, California Institute of Technology, 2003.

[10] L. Tsai. Robot Analysis: The Mechanics of Serial and Parallel Manipulators. Wiley, 1999

[11] R. Deiterding and S. L. Wood. Parallel adaptive fluidstructure interaction simulations of explosions impacting building structures. Computers \& Fluids, 88:719-729, 2013.

[12] J. D. Eldredge, J. Toomey, and A. Medina. On the roles of chord-wise flexibility in a flapping wing with hovering kinematics. Journal of Fluid Mechanics, 659:94-115, 9 2010 .

TABLE 6. RELATIVE ERROR (\%) OF NONDIMENSIONAL MEAN FORCE AND MOMENTS

\begin{tabular}{ccccccc}
\hline & \multicolumn{3}{c}{$R e_{r}=100$} & \multicolumn{4}{c}{$R e_{r}=500$} \\
Case & $\bar{F}_{x}$ & $\bar{F}_{y}$ & $\bar{M}_{z}$ & $\bar{F}_{x}$ & $\bar{F}_{y}$ & $\bar{M}_{z}$ \\
\hline 1 & -2.59 & 3.33 & -3.85 & 3.33 & 5.45 & -3.75 \\
2 & 2.47 & 0.74 & 2.55 & 2.35 & 3.83 & -4.29 \\
3 & 1.27 & 0.45 & 0.72 & 2.31 & 4.65 & -3.43 \\
4 & 4.86 & 4.28 & 3.54 & 3.51 & 2.37 & -2.32 \\
5 & 4.83 & 0.47 & 0.25 & 4.34 & 4.39 & -2.67 \\
6 & 2.10 & 3.19 & 1.52 & 3.00 & 1.82 & -3.96 \\
7 & 1.41 & 0.99 & 3.28 & 4.31 & 2.32 & -3.07 \\
\hline
\end{tabular}

TABLE 7. RELATIVE ERROR (\%) OF NONDIMENSIONAL PEAK FORCE AND MOMENTS

\begin{tabular}{ccccccc}
\hline & \multicolumn{3}{c}{$R e_{r}=100$} & \multicolumn{3}{c}{$R e_{r}=500$} \\
Case & $\bar{F}_{x}$ & $\bar{F}_{y}$ & $\bar{M}_{z}$ & $\bar{F}_{x}$ & $\bar{F}_{y}$ & $\bar{M}_{z}$ \\
\hline 1 & 4.40 & 5.07 & -3.66 & 4.40 & 3.98 & -4.17 \\
2 & 4.46 & 2.42 & 2.62 & 2.72 & 4.33 & -2.34 \\
3 & 4.20 & 3.20 & 4.80 & 3.32 & 2.68 & -4.59 \\
4 & 4.67 & 2.22 & 3.71 & 0.18 & 2.51 & -2.85 \\
5 & 3.57 & 3.37 & 1.26 & 4.09 & 4.97 & -3.63 \\
6 & 2.04 & 3.08 & 1.52 & 3.92 & 2.08 & -4.44 \\
7 & 2.20 & 1.91 & 2.26 & 3.29 & 3.79 & -4.40 \\
\hline
\end{tabular}



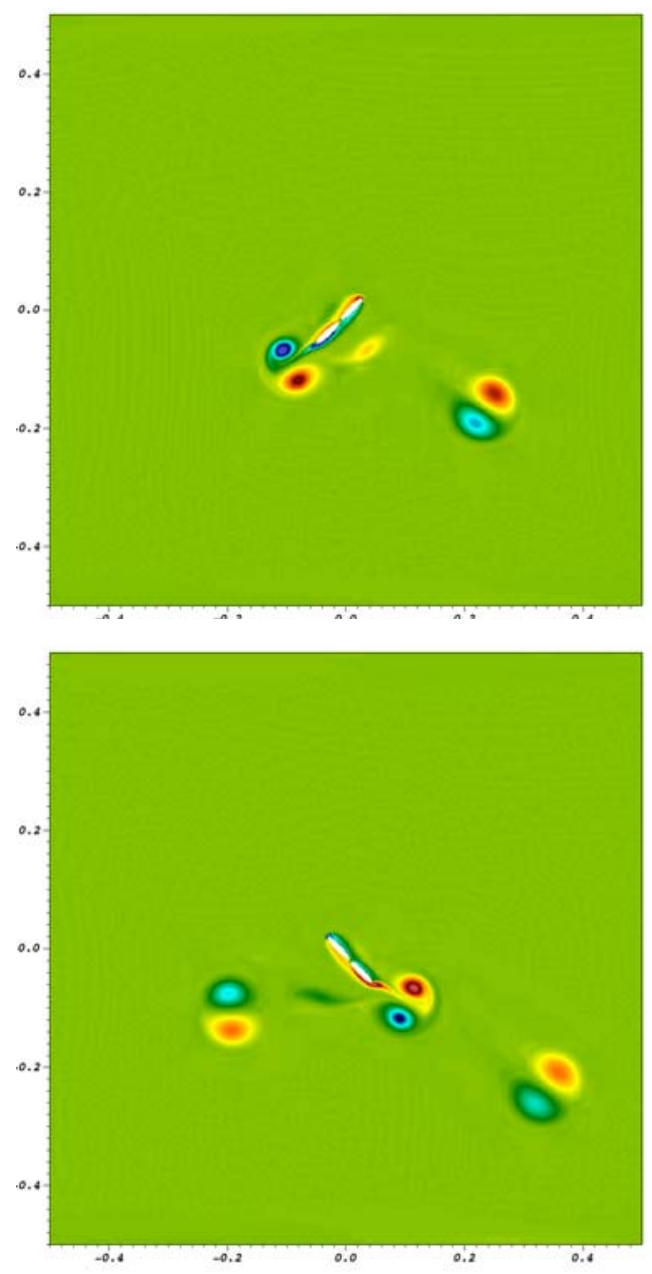

FIGURE 4. CASE $2.2 \sigma_{t}=1.85, \sigma_{r}=1.885, \Phi=0 R e_{r}=100$ : VORTICITY AT $t / \tau_{r}=1.05$ (LEFT), 1.58 (RIGHT).

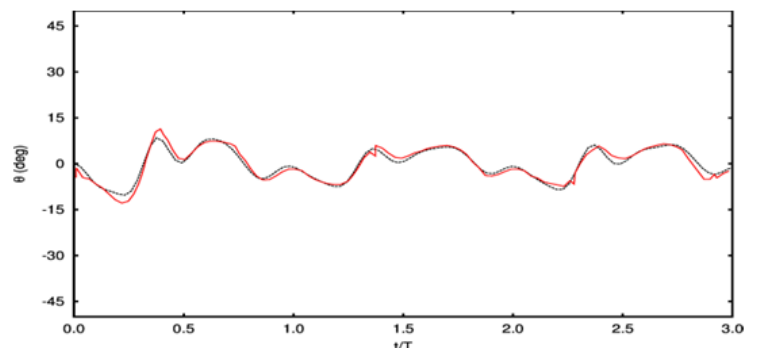

FIGURE 7. CASE $1 \sigma_{t}=0.628, \sigma_{r}=0.628, \Phi=0:$ HINGE DEFLECTION ANGLE OVER TIME. EXPERIMENTAL RESULTS (-); CURRENT (- -).
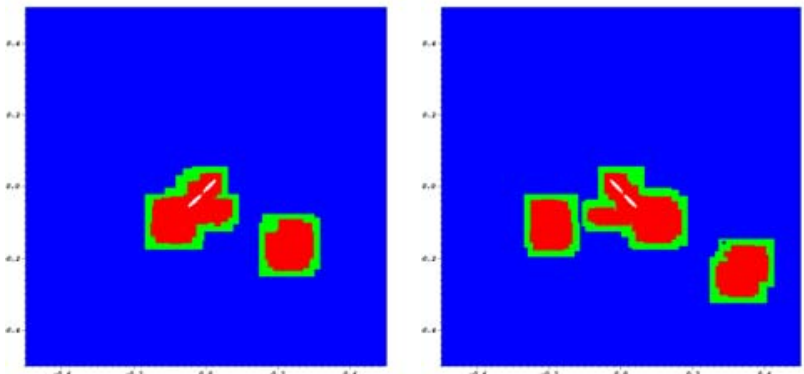

FIGURE 5. CASE $2.2 \sigma_{t}=1.85, \sigma_{r}=1.885 \Phi=0 R e_{r}=100: 3$ REFINEMENT LEVELS (INDICATED BY COLOR) AT $t / \tau_{r}=$ 1.05(LEFT), 1.58(RIGHT).
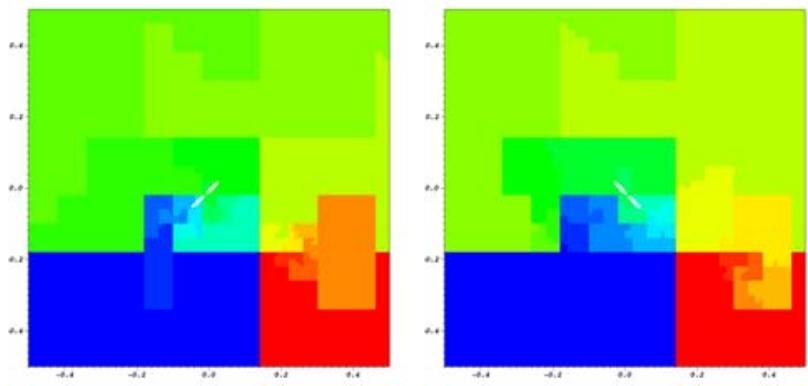

FIGURE 6. CASE $2.2 \sigma_{t}=1.85, \sigma_{r}=1.885 \Phi=0 R e_{r}=100$ : DOMAIN DISTRIBUTIONS TO 24 PROCESSORS (INDICATED BY COLOR) AT $t / \tau_{r}=1.05(\mathrm{LEFT}), 1.58$ (RIGHT).

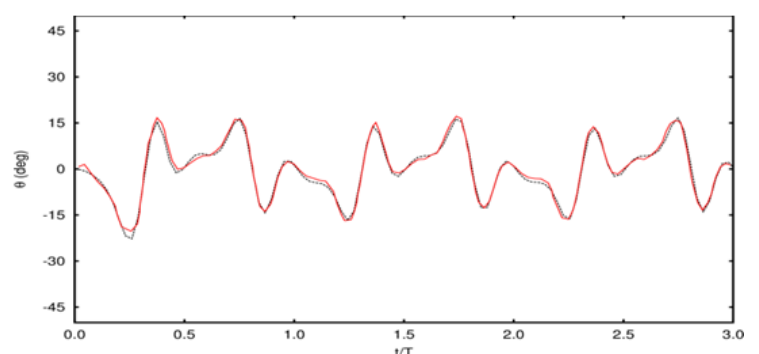

FIGURE 8. CASE $2 \sigma_{t}=1.85, \sigma_{r}=1.885, \Phi=0$ : HINGE DEFLECTION ANGLE OVER TIME. EXPERIMENTAL RESULTS $(-)$; CURRENT (- -).

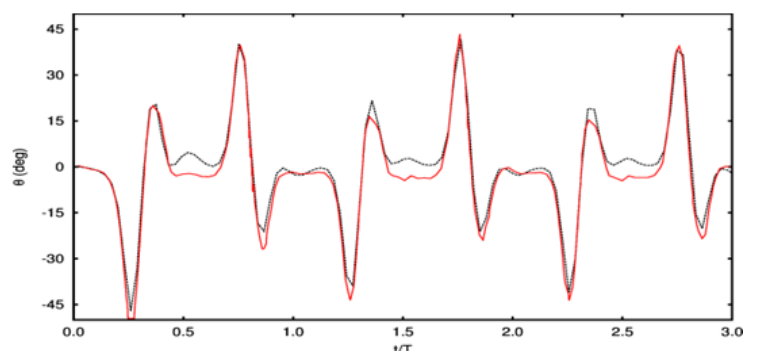

FIGURE 9. CASE $4 \sigma_{t}=3.770, \sigma_{r}=3.770, \Phi=0:$ HINGE DEFLECTION ANGLE OVER TIME. EXPERIMENTAL RESULTS $(-)$; CURRENT (- -). 